\title{
Causal Beliefs of Schizophrenia among sample of Iraqi Schizophrenic Inpatients' Families in Iraq
}

\author{
Shalan J.R. Al-Abbudi FIBMS \\ Section of Psychiatry, Dept. of Medicine, Al-Imamein Al-Kadhimein Medical City, Baghdad, Iraq
}

\begin{abstract}
Background Schizophrenia is a debilitating mental illness that affects $1 \%$ of the population in all cultures.

Objective To investigate causal beliefs regarding schizophrenia among families of Iraqi schizophrenic inpatients, and discuss the differences and similarities across cultures.

Methods Two hundred Iraqi schizophrenic inpatients fulfilling DSM-IV criteria of schizophrenia were included. Causal beliefs of their key relatives were examined. Information list including open question of the causal beliefs of schizophrenia and sociodemographic data was used. The statistical significance of the findings was tested.

Results Key relatives attributed natural causes to schizophrenia more often than supernatural causes. Stress was $44 \%$. Stresses were related to life events, trauma, social problems, war, prison and poverty. Other causes were: $27 \%$ organic and $15 \%$ personality attributions. Supernatural causal beliefs were only $29 \%$, including witchcraft, envy, possession, devil, karama and wish or punishment of God.

Conclusions The major causal beliefs of schizophrenia amongst Iraqi relatives of the studied sample were stresses. Families' attribution of supernatural causes was similar across cultures. Organicity was more in the European studies.

Key Words Schizophrenia, causes, beliefs, families, Iraq.
\end{abstract}

\section{Introduction}

chizophrenia is a debilitating mental illness that affects $1 \%$ of the population in all cultures. It affects equal numbers of men and women, but the onset is often later in women than in men ${ }^{(1)}$. Exploring what relatives of patients who have schizophrenia believe about the causes and the psychosocial consequences of the disorder has been claimed to be useful in appraising patients' family environment and planning psychosocial interventions ${ }^{(2,3)}$.

Murdock et al ${ }^{(4)}$ and Minas et al ${ }^{(5)}$ drew together causal beliefs from 139 traditional and contemporary societies from the World Ethnographic Atlas, identifying two broad constructs, natural and supernatural, and a variety of sub-constructs within each of these. These included, within the natural group, causes such as stress, infection, and organic deterioration, and, within the supernatural group, causes such as fate, mystical retribution, and magical causation.

Investigating family members' causal beliefs regarding schizophrenia is an important step in the management of the illness because it may influence the help-seeking pathway of individuals with schizophrenia ${ }^{(6)}$. It is a widely shared belief that an increase in the public's mental health literacy ${ }^{(7,8)}$ will result in an improvement of attitudes towards people with mental illness. Most of the current anti-stigma programs are based on this rationale ${ }^{(7,9)}$. 
In particular, promoting biological concepts as a causal explanation for mental disorders is considered to be a promising strategy. The argument is that if the causes of mental disorders were attributed to factors outside the individuals' control, people's reactions to those with mental illness would be less negative. German study concluded; one has to say that we are facing a dilemma. On the one hand, there are good reasons for improving the public's mental health literacy by informing them about the views shared by mental health professionals on the etiology of schizophrenia, as they may have a positive effect on people's readiness to seek professional help ${ }^{(7,10)}$.

On the other, promulgating biological factors as a cause of the disorder may lead to more instead of less rejection (7) Family members often provide psychosocial support and assistance in seeking treatment for individuals with schizophrenia. It is crucial to understand what family members believe to be the causes of schizophrenia, as this likely influences the family's help-seeking decisions ${ }^{(11)}$.

The aim of this study is to find out the causal beliefs regarding schizophrenia among schizophrenic inpatients' families in Iraq, and compare the results with several other cultural studies.

\section{Methods}

\section{Design and setting}

This is a cross-sectional study with analytic component. It was conducted in Ibn-Rushd Psychiatric Teaching Hospital, Baghdad, Iraq. The data collection was done during the period $1^{\text {st }}$ Aug. 2009 to $30^{\text {th }}$ Apr. 2010.

\section{Study Population and Sampling}

Assessment of 200 schizophrenic inpatients diagnosed by senior psychiatrists as acute schizophrenia either first episode or in relapse, was done

\section{Inclusion criteria}

All met the DSM-IV diagnostic criteria for schizophrenia.

\section{Exclusion criteria}

Current serious or unstable medical illness history of seizure disorder; history of multiple adverse drug reactions; current substance abuse, and pregnant patients were excluded. All patients assessed and followed up by full mental state examination. Families were assessed for the causes of the illness from their viewpoint and information list for collection of sociodemographic data and the family beliefs about the causation of schizophrenia illness was used. The list include open question about the most likely causes of illness and only the first answer was taken for analysis.

\section{Definition of variables}

The independent variables evaluated to explain causal beliefs were socio-demographics (age, gender, marital status, economic status, and level of education), and characteristics of the disease (duration).

\section{Statistical Analysis}

Statistical package of social sciences (SPSS) version 12 was used for data entry and analysis.

\section{Ethical Issues}

After granting approval from the concerned health authorities in Baghdad, informed consent was obtained from the patients' families after clarifying the objectives of the study. Names were kept anonymous and interviews were conducted with full privacy.

\section{Results}

The sample consisted of 200 patients and 200 key relatives. One hundred and twenty two (61\%) of the patients were male. The mean \pm SD age of the patients was $32.7 \pm 9.21$ years. One hundred and eleven (57.5\%) were single. The mean age at onset $22.9 \pm 7.1$ years; $95 \%$ Muslim, 4\% christen and 1\% from other religion. About 51.5\% satisfied their living, 39\% were poor and $9.5 \%$ rich economic status.

Educational level; illiterate $8.5 \%$, primary $24.5 \%$, intermediate $29 \%$, high school $17.5 \%$, 
university and postgraduates 20.5\% (Table 1 and fig. 1).

Table 1. Frequency and percentage of sociodemographic features of schizophrenic patients

\begin{tabular}{cccc}
\hline & Feature & No. & $\mathbf{\%}$ \\
& $\mathbf{1 7 - 2 5}$ & 54 & 27 \\
Age & $\mathbf{2 6 - 3 5}$ & 79 & 39.5 \\
(years) & $\mathbf{3 6 - \mathbf { 4 5 }}$ & 43 & 21.5 \\
& $\mathbf{4 6 - 5 5}$ & 21 & 10.5 \\
& $\mathbf{5 6 - 6 5}$ & 3 & 1.5 \\
Gender & Male & 122 & 61 \\
& Female & 78 & 39 \\
& Single & 115 & 57.5 \\
Marital & Married & 38 & 19 \\
Status & Separated & 23 & 11.5 \\
& Divorced & 20 & 10 \\
& Widowed & 4 & 2 \\
Economic & Poor & 78 & 39 \\
Status & Satisfied & 103 & 51.5 \\
& Rich & 19 & 9.5 \\
& Illiterate & 17 & 8.5 \\
Education & PS & 49 & 24.5 \\
al Level & IS & 58 & 28 \\
& HS & 35 & 17.5 \\
& University and PE & 41 & 20.5 \\
\hline
\end{tabular}

PS = Primary School, IS = Intermediate School, HS = High School, $\mathrm{PE}=$ Postgraduate Education

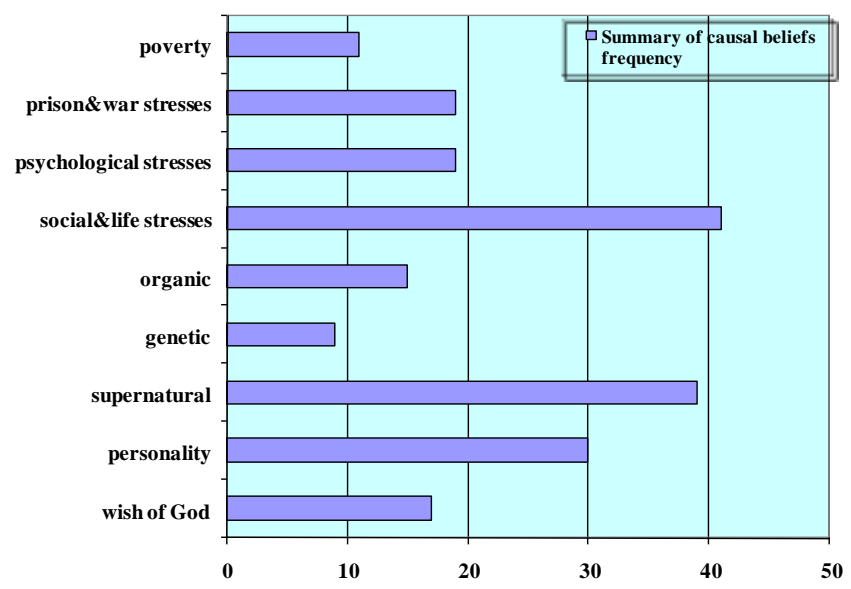

Fig. 1. Summary of the causal beliefs frequency among schizophrenic patients' families in Iraq
The mean duration of illness was $9.76 \pm 7.2$ years. The etiological beliefs were assessed from the relatives of the patients; mainly those accompanied the patients during the period of admission, it was that stress of various types represents nearly $50 \%$ of beliefs as cause of illness.

Social and life stresses (20.5\%) include family problems, love affair, study, pregnancy, and military stresses. Psychological trauma and stress (9.5\%) of the beliefs; fear, loss of close relative, death of one or both parent, and seeing dead person. War and prisons stress (9.5\%). Poverty as believed was reported by (5.5\%). Those who were attributed the illness to the organic and genetic reasons were $12 \%$. Organic related causes $(7.5 \%)$ include trauma, road traffic accidents, infection and early childhood illnesses, brain lesion, head injuries, and drug abuse. genetic causes were $4 \%$. Supernatural power attributed as the causative agent of schizophrenic process founded in 19.5\% including; devil, envy, witchcraft, and karama (locally known as sharah). Personality related causes; they marked it as him / herself, were $15 \%$. Wish or punishments of God were represented in $8.5 \%$ (Table 2 ).

Table 2. Causal beliefs frequency

\begin{tabular}{cccc}
\hline Causal Belief & & No. & $\%$ \\
\hline \multirow{2}{*}{ Wish of God } & Positive & 17 & 8.5 \\
& Negative & 183 & 91.5 \\
\hline \multirow{2}{*}{ Personality } & Positive & 30 & 15 \\
& Negative & 170 & 85 \\
\hline \multirow{2}{*}{ Supernatural } & Positive & 30 & 19 \\
& Negative & 170 & 81 \\
\hline \multirow{2}{*}{ Genetic } & Positive & 9 & 4.5 \\
& Negative & 191 & 95.5 \\
\hline \multirow{2}{*}{ Organic } & Positive & 15 & 7.5 \\
& Negative & 185 & 92.5 \\
\hline Social and life & Positive & 41 & 20.5 \\
stresses & Negative & 159 & 79.5 \\
\hline \multirow{2}{*}{ Psychological stress } & Positive & 19 & 9.5 \\
& Negative & 181 & 90.5 \\
\hline Prison and war & Positive & 19 & 9.5 \\
stress & Negative & 181 & 90.5 \\
\hline \multirow{2}{*}{ Poverty } & Positive & 11 & 5.5 \\
& Negative & 189 & 94.5 \\
\hline Total & & $\mathbf{2 0 0}$ & $\mathbf{1 0 0}$ \\
\hline
\end{tabular}




\section{Discussion}

Illness causal beliefs shape illness experience, and are important in decisions about treatment choice and treatment adherence, and in the success of the therapeutic relationship. The 9 most commonly reported causes by the relatives of the patients in the present study were social and life stress (20.5\%), supernatural power (19.5\%), personality (15\%), psychological stress (9.5\%), war and prison stress $(9.5 \%)$, wish of God (8.5\%), organic (7.5\%), poverty $(5.5 \%)$ and genetic $(4.5 \%)$. . Findings were significant when compare the presence or absence of each cause among the total causes (Table2).

These beliefs were categorized to 3 main groups; stress 44\%, supernatural $29 \%$ and organic $27 \%$. The Indian study show the majority of patients (70\%) considered spiritual and mystical factors as the cause of their predicament; $22 \%$ held multiple models of illness ${ }^{(12)}$. Nigerian study show the single most important etiological factors were that "it is Satan's work" (35.8\%) and "it is a natural illness" (23.2 \%). Other factors were "genetic" (9.5\%), "witchcraft" (10.5\%) and "curse by enemies" $(10.5 \%)^{(13)}$.

Causative models are influenced by available knowledge and practices in the culture ${ }^{(13)}$. The African American study show the 5 most commonly reported causes were disturbance of brain biochemistry (49.6\%), drug/alcohol abuse $(42.5 \%)$, hereditary factors $(40.9 \%)$, brain injury $(40.2 \%)$, and avoidance of problems in life $(37.8 \%)$. The mean number of likely or very likely causes endorsed by participants was $7.5+/-5.7^{(14)}$. Some $47.9 \%$ reported one or more esoteric factors as a cause. Of the 6 esoteric factors, possession by evil spirits $(28.3 \%)$, radiation $(20.2 \%)$, and punishment by God (19.7\%) were most common. Esoteric causes were more commonly chosen by male participants, those with 12 years of education or less, and participants who reported never having known someone with schizophrenia ${ }^{(14)}$.
Bali study explore that the key relatives attributed supernatural causes to schizophrenia more often than natural causes. Compared with relatives who listed a natural cause as most important (14 relatives, or 36 percent), relatives who considered a supernatural cause as being the most important ( 25 relatives, or 64 percent) had a significantly higher mean age and less education and were more likely to have family members with schizophrenia who had never received psychiatric medical treatment ${ }^{(15)}$. Italian study show only 24 percent of the relatives (156 of 652) believed that both biological and psychosocial factors had been involved in the development of their loved ones' schizophrenia. Seventy percent of the relatives (457 of 652) maintained that the disorder was due exclusively to psychosocial factors, such as stress, psychological traumas, or the breakdown of a romantic relationship; 6 percent (39 of 652) thought that the disorder had been caused exclusively by biological factors. Patients' intentional behaviors, such as using drugs or keeping bad company, were mentioned as factors by 28 percent of the relatives (182 of 652) ${ }^{(16)}$.

Another Indian study show supernatural cause was named by only $12 \%$ of the families and as the only cause by $5 \%$. Psychosocial stress was most commonly cited cause, followed by personality defect and heredity. A small number of families (14\%) could not name any cause and 39\% named more than one cause ${ }^{(17)}$. German study to examine how the German public's causal attributions of schizophrenia and their desire for social distance from people with schizophrenia found at 2001; Brain disease $70.0 \%$, Heredity $60.2 \%$, Life event $72.3 \%$, Stress at work $57.7 \%$, Broken home $39.3 \%$, Lack of parental affection $30.4 \%$, Lack of willpower $36.4 \%$, and Immoral lifestyle $20.9 \%{ }^{(7)}$.

This study shows that Iraqi people explore the dilemma of stress in its various components as the major causative factor of schizophrenia (44\%). Majority of stresses were related to life events, trauma, social problems, war, prison and poverty. Beliefs of stress were not related 
to the current critical situation in Iraq since the mean duration of illness is $9.76 \pm 7.2$ years, but may be related to that before 2003. Kaplan and Sadock ${ }^{(19)}$, Barlow and Durand ${ }^{(20)}$, and Sue et al ${ }^{(18)}$ explain the etiology of schizophrenia as; Stress Diathesis Model.

This model postulates that a person may have a specific vulnerability that when acted on by some stressful environmental influence, allows the symptoms of schizophrenia to develop. In the most general stress-diathesis model, the diathesis or the stress can be biological or environmental or both. Supernatural factors were not deferred widely from our study in comparison with the above studies, indicates that Iraqis were of similar thinking about the causal beliefs of schizophrenia with Asian, European, African and American people. Across cultural similarity of traditional beliefs were present like witchcraft, envy, possession, devil, karama and wish or punishment of God. Organic beliefs of schizophrenic etiology founded less in our study and Asian and African studies in comparison with the European and American studies.

In conclusion, beliefs about stress, societal, family, personality and esoteric causes in this clinical sample indicate the need for improved psychoeducation of the community at large, there is a need for public mental health literacy and welfare support to actualize the potential of families to play useful community psychosocial roles. Future research should seek to better understand how esoteric beliefs about causation affect attitudes toward people with mental illnesses and acceptance of mental health treatment by those individuals.

\section{Acknowledgment}

The author consulted Dr. Manaf Al-Jadri, consultant psychiatrist, College of Medicine, Jordan University.

\section{Conflict of interest}

None.

\section{Funding}

None.

\section{References}

1. Schultz SH, North SW, Shields CG. Schizophrenia: A Review. Am Fam Physic. 2007; 75(12): 1821-9.

2. Atkinson JM, Coia DA. Families Coping With Schizophrenia: A Practitioner's Guide to Family Groups. Chichester, England, Wiley, 1995.

3. Robinson E. Causal attributions about mental illness: relationship to family functioning. Am J Orthopsychiat. 1996; 66: 282-95.

4. Minas $H$, Klimidis $S$, Tuncer $C$. Illness causal beliefs in Turkish immigrants. BMC Psychiat. 2007; 7: 34. DOI: 10.1186/1471-244X-7-34

5. Murdock GP, Wilson SF, Frederick V. World distribution of theories of illness. Transcultural Psychiat Res Rev. 1980; 17: 37-64.

6. Phillips MR, Li Y, Stroup $S$, et al. Causes of schizophrenia reported by patients' family members in China. Br J Psychiat. 2000; 177: 20-5.

7. Angermeyer MC, Matshinger $\mathrm{H}$. Causal beliefs and attitudes to people with schizophrenia. Trend analysis based on data from two population surveys in Germany. Br J Psychiat. 2005; 186: 331-4.

8. Jorm AF. Mental health literacy: public knowledge and beliefs about mental disorders. $\mathrm{Br} J$ Psychiat. 2000; 177: 396-401.

9. Lopez-lbor JJ. The WPA and the fight against stigma because of mental illness. World Psychiat. 2002; 1 : 30-1.

10. Angermeyer MC, Matschinger $H$, Riedel-Heller SG. Whom to ask for help in case of mental disorder? Preferences of the lay public. Soc Psychiat Psychiat Epidemiol. 1999; 34: 202-10.

11. Esterberg ML, Compton MT. Causes of schizophrenia reported by family members of urban African American hospitalized patients with schizophrenia. Compr Psychiat. 2006; 47: 221-6.

12.Saravanan B, Jacob KS, Johnson $S$, et al. Belief models in first episode schizophrenia in South India. Soc Psychiat Psychiat Epidemiol. 2007; 42: 446-51.

13. Ohaeri JU, Fido AA. The opinion of caregivers on aspects of schizophrenia and major affective disorders in a Nigerian setting. Soc Psychiat Psychiat Epidemiol. 2001; 36(10): 493-9.

14. Compton MT, Esterberg ML, Broussard B. Causes of schizophrenia reported by urban African American lay community members. Compr Psychiat. 2008; 49: 87-93.

15. Kurihara $T$, Kato $M$, Reverger $R$, et al. Beliefs about causes of schizophrenia among family members: a community-based survey in Bali. Psychiat Serv. 2006; 57(12): 1795-9.

16. Magliano L, Guarneri M, Fiorillo $A$, et al. $A$ multicenter Italian study of patients' relatives' beliefs about Schizophrenia. Psychiat Serv. 2001; 52: 152830.

17. Srinivasan TN, Thara R., Beliefs about causation of schizophrenia: do Indian families believe in 
Al-Abbudi, Causal Beliefs of Schizophrenia ....

supernatural causes? Soc Psychiat Psychiat Epidemiol. 2001; 36: 134-40.

18. Sue D, Sue D, Sue S. Understanding abnormal behaviour. Boston: Houghton Mifflin Compan; 1994.

19. Kaplan HI, Sadock BJ. Synopsis of psychiatry: Behavioural sciences Clinical psychiatry. $8^{\text {th }}$ ed. New York: Lippincott Williams \& Wilkins; 1998.
20. Barlow DH, Durand VM. Abnormal psychology. Monterey CA: Brookes: Cole Publishing Company; 1995.

Email: shalanjoodah@yahoo.com

Received: $30^{\text {lst }}$ Dec. 2015: Accepted $18^{\text {th }}$ Apr. 2016 